\title{
Three-dimensional quantitative evaluation method of nonrigid registration algorithms for adaptive radiotherapy
}

\author{
B. Rodriguez-Vila ${ }^{\text {a) }}$ \\ Bioengineering and Telemedicine Group, Technical University of Madrid, Madrid 28040. Spain and \\ Networking Research on Bioengineering. Biomaterials and Nanomedicine (CIBER-BBN). Madrid. Spain \\ F. Gaya \\ Bioengineering and Telemedicine Grolap, Technical University of Madrid, Madrid 28040. Spain \\ and Section of Biostatistics, La Paz University Hospital, Madrid 28046, Spain \\ F. Garcia-Vicente \\ Department of Radiotherapy, University Hospital of La Princesa, Madrid 28006, Spain \\ and Department of Medical Physics. University Hospital of La Princesa. Madrid. Spain \\ E. J. Gomez \\ Bioengineering and Telemedicine Group. Technical University of Madrid, Madrid 28040, Spain and \\ Networking Research on Bioengineering. Biomaterials and Nanomedicine (CIBER-BBN). Madrid. Spain
}

(Received 24 July 2009; revised 21 December 2009; accepted for publication 30 December 2009; published $\mathrm{xx} \times \mathrm{xx} \mathrm{xxx}$ )

Purpose: Current radiotherapy is progressing to the concept of adaptive radiotherapy, which implies the adaptation of planning along the treatment course. Nonrigid registration is an essential image processing tool for adaptive radiotherapy and image guided radiotherapy, and the threedimensional (3D) nature of the current radioherapy techniques requires a 3D quantification of the registration error that existing evaluation methods do not cover appropriately. The authors present a method for 3D evaluation of nonrigid registration algorithms' performance, based on organ delineations, capable of working with near-spherical volumes even in the presence of concavities.

Methods: The evaluation method is composed by a volume shape description stage, developed using a new $a d$ hoc volume reconstruction algorithm proposed by the authors, and an error quantification stage. The evaluation method is applied to the organ delineations of prostate and seminal vesicles, obtained by an automatic seginentation method over images of prostate cancer patients treated with intensity modulated radiation therapy.

Results: The volume reconstruction algorithm proposed has been shown to accurately model complex 3D surfaces by the definition of clusters of control points. The quantification method, inspired by the Haussdorf-Chebysev distance, provides a measure of the largest registration error per control direction, defining a valid metric for concave-convex volumes. Summarizing, the proposed evaluation methodology presents accurate results with a high spatial resolution in a negligible computation time in comparison with the nonrigid registration time.

Conclusions: Experimental results show that the metric selected for quantifying the registration error is of utmost importance in a quantitative evaluation based on measuring distances between volumes. The accuracy of the volume reconstruction algorithm is not so relevant as long as the reconstruction is tight enough on the actual volume of the organ. The new evaluation method provides a smooth and accurate volume reconstruction for both the reference and the registered organ, and a complete 3D description of nonrigid registration algorithms' performance, resulting in a useful tool for study and comparison of registration algorithms for adaptive radiotherapy.

Key words: non-rigid registration, 3D quantitative evaluation, adaptive radiotherapy

\section{INTRODUCTION}

Nonrigid image registration is an essential tool for the development of adaptive radioherapy, since it allows carrying out morphological comparisons in the presence of anatomic variations for therapy monitoring. ${ }^{1}$ It is a more general approximation than widely used methods such as rigid or affine registration, but it requires a more complex methodology and a larger computational effort. ${ }^{2}$

Several nonrigid registration algorithms have been developed ${ }^{3}$ and its application on adaptive radiation therapy tested. ${ }^{4-8}$ However, the evaluation of their performance is especially difficult since point-to-point correspondence between both images is usually unknown. A real gold standard to evaluate the performance of the algorithms does not exist. Previous efforts to objectively evaluate and compare the performance of image registration algorithms using standard evaluation criteria have been made. There are some general projects focused on validation of nonrigid registration algorithms. Fitzpatrick et al. ${ }^{9}$ centered their efforts on the evalu- 
ation of multimodal rigid registration accuracy, while Barillot et al. ${ }^{10}$ worked on intersubject brain registration. "NIREP,"11 led by Christensen tried to cover all the range of registration algorithms. Other validation tools for threedimensional (3D) objects' segmentation such as "Valmet,"12 led by Gerig, are used for evaluation of nonrigid registration algorithms.

The three-dimensional nature of the radiotherapy process requires a full $3 \mathrm{D}$ description of the variations in the volume segmentations in order to localize the regions where manual or automatic segmentations have more differences and to compute the range of these variations in each region. Thus, the value of a distance or an overlapped volume is not enough. Remeijer et al. ${ }^{13}$ approached the problem through a general methodology for the analysis of the variations in the delineations developed by various specialists on multimodal images although the method presents an important limitation: It is useful only for convex volumes, so realistic reconstructions of the organs are excluded. Mageras et al. ${ }^{14}$ drew inspiration from this past work to evaluate a registration algorithm applied over lung cancer images, defining a new metric that allows measuring the error on convex-concave volumes. The metric proposed measures the furthest (but not necessarily the largest) error from the center of reference for every control direction. Finally, Foskey et al. ${ }^{15}$ applied a similar method to evaluate a registration algorithm applied over prostate cancer images.

The aim of this research work is to improve the previously mentioned methodologies for quantitative evaluation of nonrigid image registration algorithms' performance, ${ }^{13-15}$ achieving an accurate measure of the registration error for both convex and concave-convex volumes and describing realistic reconstructions of human organs.

\section{MATERIALS AND METHODS}

The evaluation methodologies used by Mageras ${ }^{14}$ and Foskey ${ }^{15}$ are inspired by the methodology proposed by Remeijer, ${ }^{13}$ and follow the same structure: A volume shape description stage, where the volume of the reference and the registered organs are reconstructed from the axial contours and quantified in terms of scalar samples of the 3D volume; and an error quantification stage that measures the distance between both reconstructions using a predefined metric.

Nevertheless, only the original work explains completely the methodology, describing both stages, so it will be the one taken as reference. In this paper we describe our proposed methodology, composed by an innovative $3 \mathrm{D}$ reconstruction algorithm and a new error metric.

\section{II.A. Volume shape description}

\section{II.A.1. Frame of reference}

The general methodology proposed in Ref. 13 covers intermodality, interspecialist, and intraspecialist error, offering a statistical model of each component. The evaluation methodologies used in Refs. 14 and 15 and also the one presented in this paper are proposed for evaluating the performance of automatic algorithms applied over the same patient during a

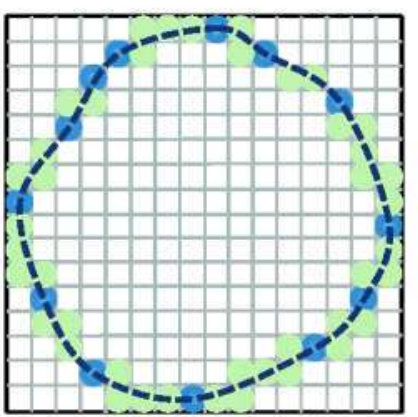

(a)

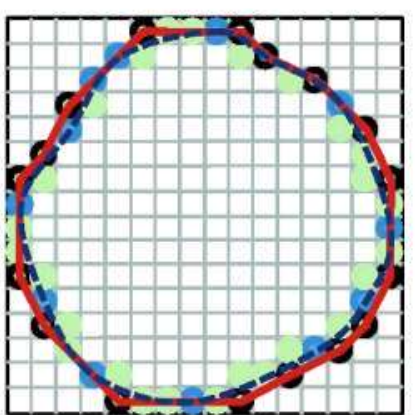

(b)
FIG. 1. (a) The original contour, formed by the darkest points, is interpolated in order to obtain a maximum variation of 1 pixel in both the horizontal and the vertical axis. The positions of these new points are rounded off, finding the locations of the pixels crossed by the contour (lightest points). (b) The automatic landmarks, represented by black circles, are extracted by eliminating redundant information.

treatment, using the same image modality, thus making easier the definition of the frame of reference.

The bones of the patient define a solid and static frame of reference but, despite the efforts of the radiotherapy technicians, the reproducibility of the positioning of a patient in each session is not perfect. Therefore a rigid registration of the bony structures is applied in order to obtain a common frame of reference for all the patient's images.

\section{II.A.2. Preprocessing}

The contours of the organs of interest in radiotherapy are described in each axial slice by manual landmarks linked by cubic interpolation. Since the current version of our reconstruction algorithm only works using linear interpolation, some automatic landmarks are computed in order obtain a new set of contours as similar as possible to the original contours. The whole process can be explained using Fig. 1. The original contour, formed by the points filled in blue, is interpolated in order to obtain a maximum variation of 1 pixel in the horizontal axis and the vertical axis. The positions of these new points are rounded off, finding the locations of the pixels crossed by the contour (points filled in green). Finally, the automatic landmarks (points outlined by black circles) are extracted by eliminating redundant information. Future developments of the algorithm will include cubic interpolation, and the preprocess step will be unnecessary.

Moreover, the organ's center of gravity is computed by averaging the position of the points of the volume enclosed by the contours.

\section{II.A.3. Organ shape description}

Volume shape is quantified in terms of scalar samples of the $3 \mathrm{D}$ volume. Therefore, the target volume is quantified by sampling the distance between a center of reference, beneath the organ's surface, and the surface of the organ for a large number of control directions defined by a uniform latitude and longitude division. If the number of directions is sufficient, the distance between samples of the surface will be 
enough to provide an adequate description of the organ. This methodology is useful for near-spherical volumes such as some of the human organs: Brain, heart, bladder, prostate, lungs, kidneys, liver, etc. On the oher hand, the description of tubular structures such as vessels, intestines, or long bones would require the description reference axis instead of a center of reference.

In order to know the number of control directions needed in each case the relation between them and the spatial resolution obtained has been computed. Let us suppose longitude $\omega$, latitude $\varphi$, and two adjoining points separated by an increment in angle $\delta$, described by the radius $r 1$ and $r 2$, and by a distance $d$ (or spatial resolution). In the case of a latitude increase, the values $\omega$ and $\varphi$ are irrelevant for the solution of the problem; but in the case of a longitude increase, keeping the latitude value constant, the value $\varphi$ affects to the solution. Solving the triangle problem formed by both radius and the segment $d$ that linked both, we obtained a formula for the increment in angle.

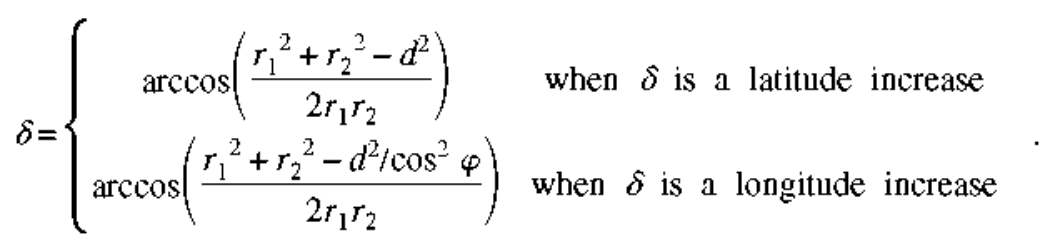

Defining $r$ as the average radius, and $\Delta=r 1-r 2$, a new definition of the increment in angle is obtained,

$$
\delta=\left\{\begin{array}{c}
\arccos \left(\frac{r^{2}+\Delta^{2} / 4-d^{2} / 2}{r^{2}-\Delta^{2} / 4}\right) \\
\arccos \left(\frac{r^{2}+\Delta^{2} / 4-d^{2} / 2 \cos ^{2} \varphi}{r^{2}-\Delta^{2} / 4}\right)
\end{array} \text { when } \delta \text { is a latitude increase } \delta\right. \text { is a longitude increase }
$$

The number of control directions $N=360 \times 180 / \delta^{2}$ is well defined, while the argument of the arccosines is greater than zero. This is achieved in any case since $d$ is always greater than $\Delta$, as well as it tends to zero when $\varphi$ tends to $\pm \pi / 2$.

The proposed algorithm, ${ }^{16}$ named "Origami," is based on the folding of space, like a piece of paper in the Japanese ancient art of origami, and consists on the combination of two bidimensional analyses of the volume of the organ instead of a single 3D one. The result of the reconstruction is a cluster of control points above the surface of the organ. It is composed of the following main steps:

- All contours drawn on axial slices are represented on a single plane and intersected by a set of control directions defined by a $\theta$ angle, centered on the projection of the point of reference on said plane [see Fig. 2(a)]. The intersections are fast and efficiently computed by solving the line-segment problem in $2 \mathrm{D}$ for every contour and every control direction. As a result, the dimensionality of the problem is reduced and the current $2 \mathrm{D}$ frame of reference is homogeneously divided into polar coordinates.

- A set of contours perpendicular to the axial ones (called radial contours in order to distinguish both types) is constructed in order to obtain a description of the volune in the interslice gaps. First, the intersection points between each control direction and every axial contour, which are described in 2D only, are raised to the height of the axial contour they belong to, adding a third co- ordinate for each point and thus returning to a $3 \mathrm{D}$ problem. Each radial contour is associated to the intersections of a $\theta$ angle and its opposite $\left(180^{\circ}+\theta\right)$ [see Fig. 2 (b)]. This step connects every axial slice of the volume with its neighbors, creating a very smooth and realistic volume without eliminating any sinall concavities of the organ. It is equivalent to the rendering of other methods but does not compute any enveloping surface.

- The new set of contours is represented on a single plane by folding down along a vertical axis sited on the center of reference [see Fig. 3(a)], reducing again the dimensionality to a $2 \mathrm{D}$ frame of reference. Next, the radial contours are intersected by a new set of radial rays defined by a $\varphi$ angle [see Fig. 3(b)] and centered on the projection of the center of reference on the plane.

- The volumetric coordinates of every surface point are extracted from the $\theta$ angle of the radial plane, and the $\varphi$ angle and $\rho$ from the set of radial rays.

\section{II.B. Error quantification}

The metric proposed is inspired by the HaussdorfChebysev metric, which defines the largest difference between two contours. Given two contours $A$ and $B$, the minimum distance between each point in $A$ and every point of contour $B$ is computed in the first place. The maximum of 

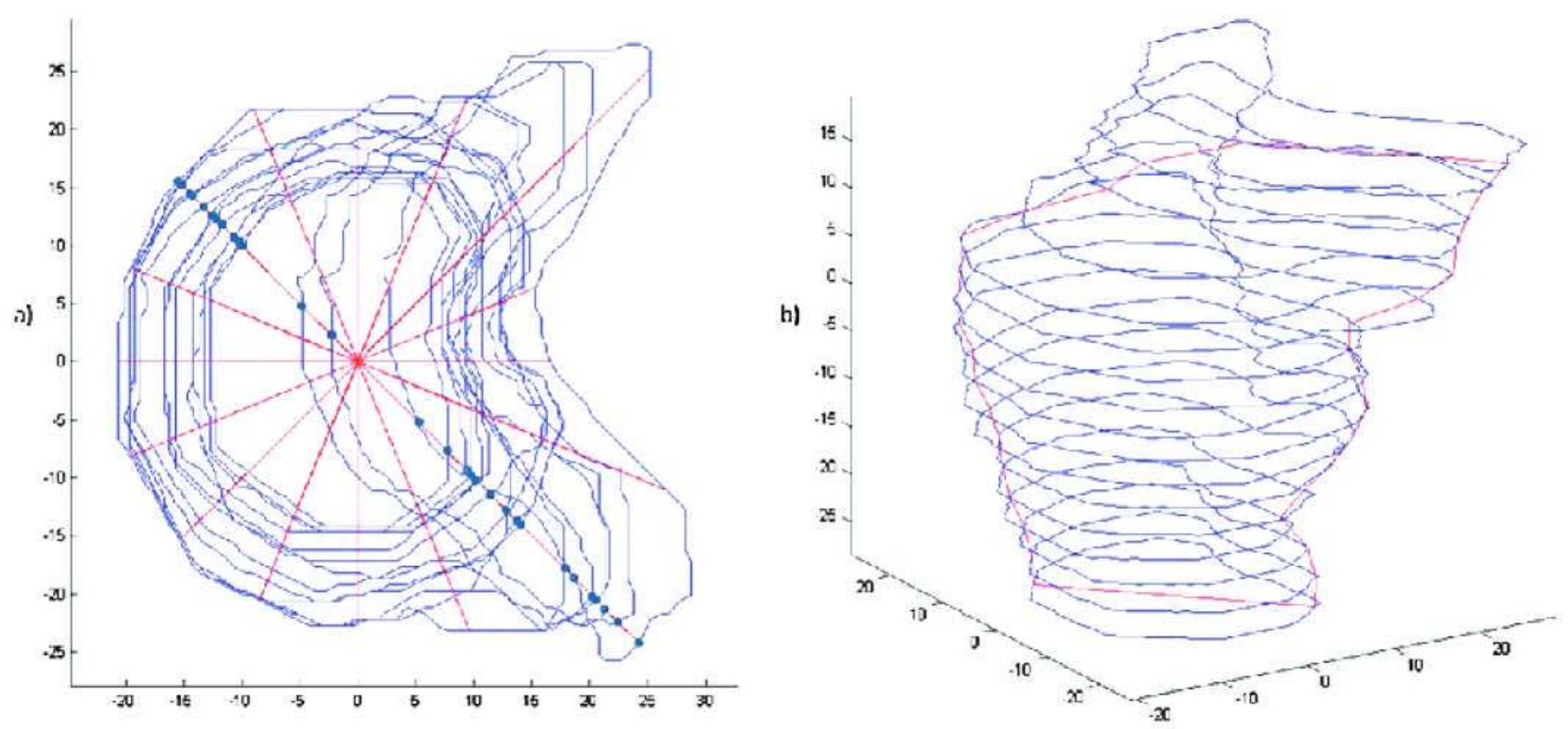

FIG. 2. (a) 2D projection of the axial contours of a prostate and seminal vesicles and the intersection points of a control direction. (b) 3D representation of the axial contours and the reconstructed "radial" contour.

these values is chosen as "the worst case." The HaussdorfChebysev metric is not symmetric, therefore, $d(A, B)$ $\neq d(B, A)$, so finally, the metric is defined as $D(A, B)$ $=\max (d(A, B), d(B, A))$.

Haussdorf-Chebysev metric requires a lot of computing time since it is necessary to compare each point from one contour to every point of the other.

Since our interest is focused on a given distance value per control direction, and not a single distance value between two volumes, we have to define a new metric. Given two 3D

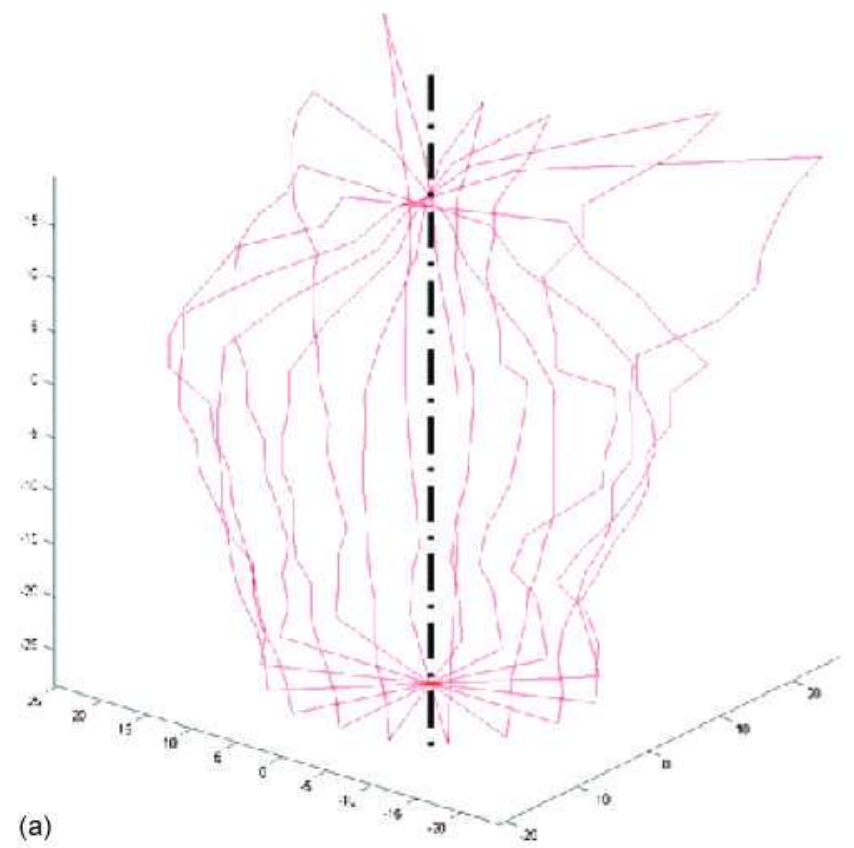

reconstructions $A$ and $B$, defined as two set of dense clusters of points over the organ's surface, the minimum distance from each point of $A$ to $B$ is easily computed finding the nearest point from $B$. This metric, as the original HaussdorfChebysev metric, is nonsymmetric, so the final metric is computed as $d_{\alpha}=\max \left(\min _{b \in B} d\left(a_{\alpha}, b\right), \min _{\alpha \in A} d\left(a, b_{\alpha}\right)\right)$ for any given control direction $\alpha$. In case that a control direction intersects a contour in more than one point, the computed distance has to take into account all the intersections (see Fig. 4).

FIG. 3. (a) 3D representation of the axial contours of the same prostate and seminal vesicles. (b) 2D projection of the radial contours and its intersection points with the control directions.

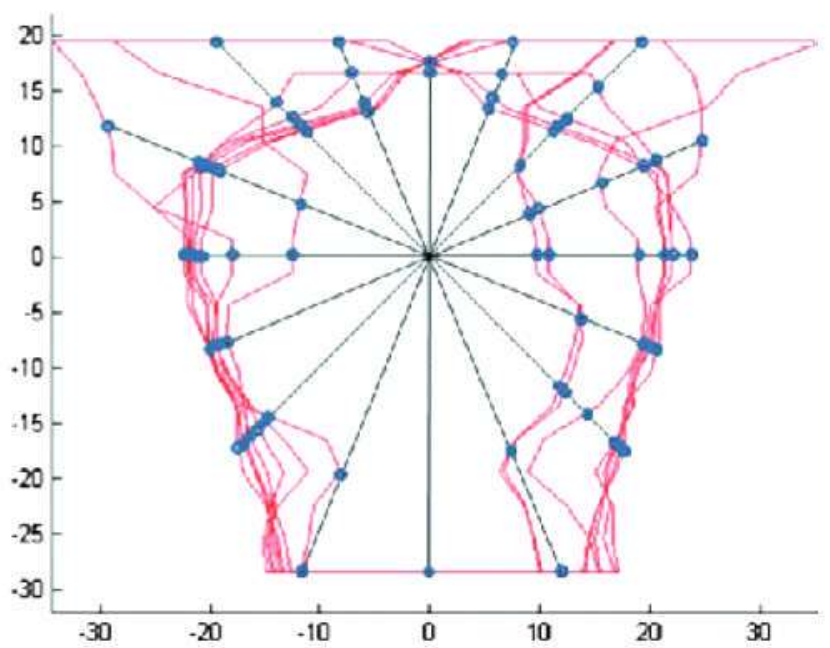

(b) 


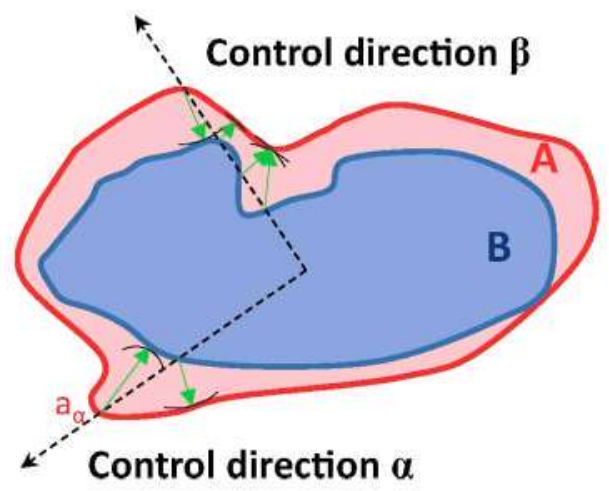

FIG. 4. Definition of the proposed error quantification metric. The error value is computed as the maximum value of the distance between the contours, measured in the intersection points between the contours and the control direction.

The proposed quantification method is similar to the method proposed in Ref. 9 for error quantification, being also insensitive to the location of the template organ's surface relative to the reference surface, but offering the maximum error value for each control direction instead of the furthest error from the center of reference. The relevance of this difference is stated in Fig. 5, where two contours of a volume, similar to a prostate and the seminal vesicles, are shown: The reference contour exhibited as a thin dotted line and the reference contour as a dashed line. The contours match remarkably well, but the concavity formed by the joint representation of the prostate and the seminal vesicles and the concavity close to the apex show small inaccuracies. In this case, the metric proposed in Ref. 14 will result in a perfect fit for both control directions since the furthest errors, measured at the furthest intersection points (pointed by white arrows), are close to zero. Nevertheless, our metric measures the distance between the volumes in every intersection point be-

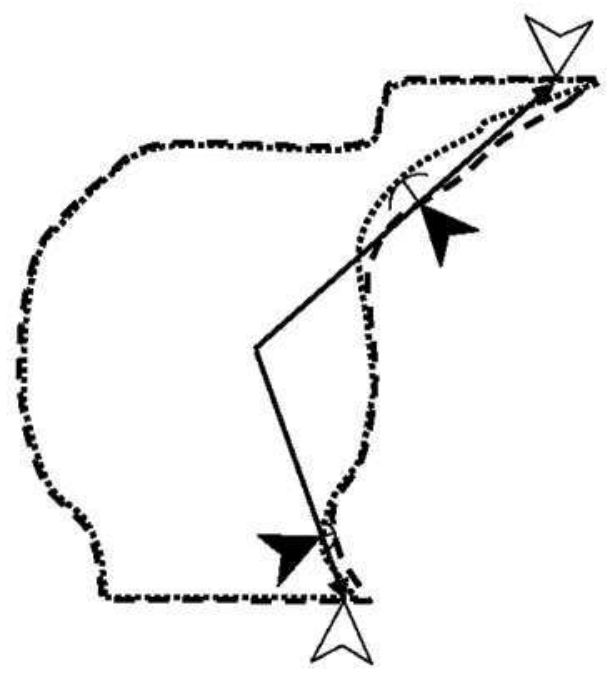

FIG. 5. Representation of a manual delineation (solid line) and an automatic delineation (dashed line) of a prostate and seminal vesicles. The furthest errors for the selected control directions are close to zero, offering a false idea of a perfect fit between delineations. The proposed metric will offer a better description of the error distribution in these cases. tween the control direction and the organs. The distances are represented by arcs with center in the intersection points, while the black arrows point at the intersection points associated to a maximum distance.

This metric, like the Haussdorf-Chebysev metric, requires a lot of computations since it compares each point of one contour to every point of the other. In order to speed up the computation process our algorithm supposes that the minimum distance between volumes for a given control direction is located between points with similar latitude and longitude. Thus, the search is restricted to a margin of $\pm 45^{\circ}$ in longitude and latitude centered in the point's coordinates, reducing the computing time to a quarter. The point of minimum distance has always been inside this margin in the cases of study.

\section{II.C. Nonrigid registration algorithm}

The evaluation methodology has been tested evaluating the results of an algorithm similar to the one used in Ref. 4. It is an intensity-based algorithm relying on the concept of optical flow proposed by Thirion. ${ }^{17}$

The deformation vector field computed by registering the anatomical images is applied to a 3D binary image formed selecting the voxels bounded by the manual segmentation of the prostate and seminal vesicles. Thus, an automatic segmentation of the organ of interest is obtained. The contours are easily extracted by selecting automatic landmarks on each slice of the transformed binary image. More specifically, the landmarks selected are the origin and the end of each straight line on every slice. Error values are computed comparing the obtained automatic delineation and a control manual one.

\section{II.D. Visualization}

The organ shape and the error measurements can be plotted over the surface of a sphere, resulting in a distance map. In this paper, distance spherical maps are represented using the equidistance cylindrical projection, showing the longitude in the horizontal axis, the latitude in the vertical axis, and the error values, in millimeters, using a color scale. Moreover, isodistance curves are added in order to facilitate the comprehension of the results.

Since the image position protocol implies that the patients are all scanned in a consistent orientation, different radial distance maps can be compared directly, and average maps can be computed point by point. Average maps using a significant number of cases will show relevant information about the performance of the registration algorithm, detecting the problematic regions.

Nonrigid registration evaluation was applied to CT images of radiotherapy treatments performed on four patients undergoing intensity modulated radiation for prostate cancer. Contours of the prostate, bladder, rectum, skin, and femoral heads were manually drawn on plan and control CT scans by the same physician, but only the prostate and seminal vesicles contours were used for evaluation of the nonrigid 


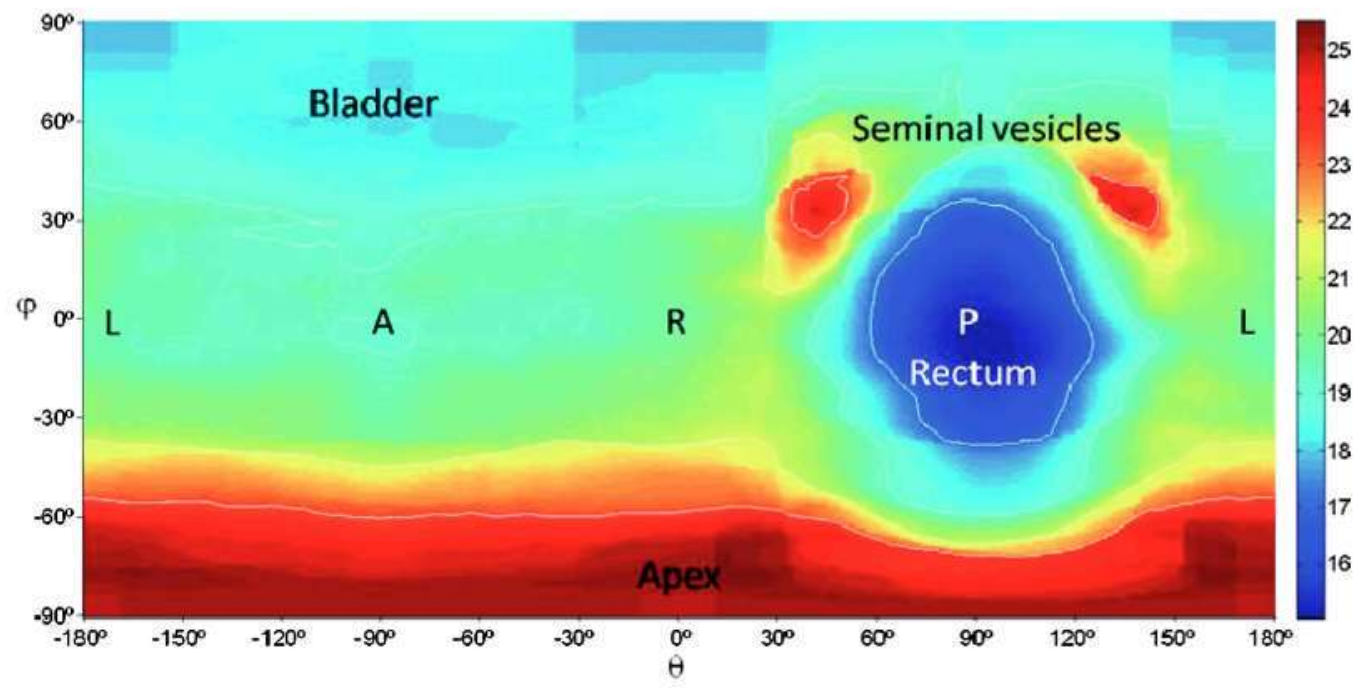

FIG. 6. Polar map representation of the surface of a prostate and seminal vesicles. The main regions and the $A-P$ axis are indicated.

algorithm performance. A representation of a mean prostate and seminal vesicles, and their enhanced principal regions, are shown in Fig. 6.

CT scans were obtained with a Toshiba Asteion CT (Toshiba Medical Systems, Japan) and the radiotherapy treatment plans were developed using a XIO 4.34.02 (Computerized Medical Systems, USA) treatment planning system. CT scans are composed by 40 slices of $512 \times 512$ pixels with a spatial resolution of $0.937 \times 0.937 \times 3 \mathrm{~mm}^{3}$, and organs of interest are around $100 \times 100 \times 20$ voxels on average.

The algorithms are developed and optimized using MATLAB R2007b and executed on a dual core $3 \mathrm{GHz}$ system with 4 GB RAM, using a 64 bit operating system.

\section{RESULTS AND DISCUSSION}

The evaluation methodology proposed, like the similar previous ones, is formed by a volume shape description stage and an error measure stage. This research work tries to improve previous results in both stages proposing a new volume reconstruction method and a new metric. This section has been divided into three subsections: Sec. III A shows the capability of the methodology of representing the common registration errors; Sec. III B compares the results of our proposed metric to the results of previous metrics, showing the distribution and magnitude of the differences between metrics; and finally, Sec. III C is focused on the reconstruction algorithm used.

Both the reconstruction algorithm and the error quantification metric proposed are highly dependent on the number of control directions and the spatial resolution desired, but also both methods are fast enough to offer accurate results in few seconds. The computing time evolution of the global evaluation method and the reconstruction algorithm are shown in Table I. The computing time of the reconstruction algorithm is linearly dependent on the number of control directions. On the other hand, the global evaluation time has quadratic dependency on the number of control directions. Results with a great spatial resolution can be computed using an angular increase of $1^{\circ}$ or $1.5^{\circ}$ in a few seconds, negligible in comparison with the registration time, which is always between 10 and $15 \mathrm{~min}$.

We have chosen an angular resolution of $\delta=1^{\circ}$ for every case, which leads to 64800 control directions. This could lead to very different spatial resolutions depending on the orientation of the surface of the organ. Where the surface's vector is almost parallel to the control direction, two contiguous points will have very similar values of radius leading to a great spatial resolution, i.e., $r 1=30.2 \mathrm{~mm}$ and $r 2=29.8 \mathrm{~mm}$ results into a spatial resolution of $0.66 \mathrm{~mm}$ in that part of the volume. On the contrary, where the surface's vector is almost perpendicular to the control direction two contiguous points may have very different values of radius, leading to a poor spatial resolution, i.e., $r 1=32 \mathrm{~mm}$ and $r 2=28 \mathrm{~mm}$ results into a spatial resolution of $4 \mathrm{~mm}$.

\section{III.A. Error visualization}

A case study has been selected as an example of common registration errors in order to highlight the advantages of the proposed methodology. Figure 7 shows the axial view (left),

TABLE I. The reconstruction time is linearly dependent on the angular resolution. On the other hand, the evaluation time has a quadratic dependency on the angular resolution.

\begin{tabular}{lccc}
\hline \hline $\begin{array}{l}\text { Angular } \\
\text { increase } \\
\delta\end{array}$ & $\begin{array}{c}\text { Number } \\
\text { of control directions }\end{array}$ & $\begin{array}{c}\text { Evaluation } \\
\text { time } \\
(\mathrm{s})\end{array}$ & $\begin{array}{c}\text { Reconstruction } \\
\text { time } \\
(\mathrm{s})\end{array}$ \\
\hline 10 & 648 & 0.231 & 0.046 \\
6 & 1800 & 0.236 & 0.048 \\
3 & 7200 & 0.365 & 0.053 \\
2 & 16200 & 0.741 & 0.073 \\
1.5 & 28800 & 1.811 & 0.081 \\
1 & 64800 & 8.236 & 0.092 \\
0.66 & 115200 & 26.955 & 0.165 \\
0.5 & 259200 & 140.371 & 0.274 \\
\hline \hline
\end{tabular}




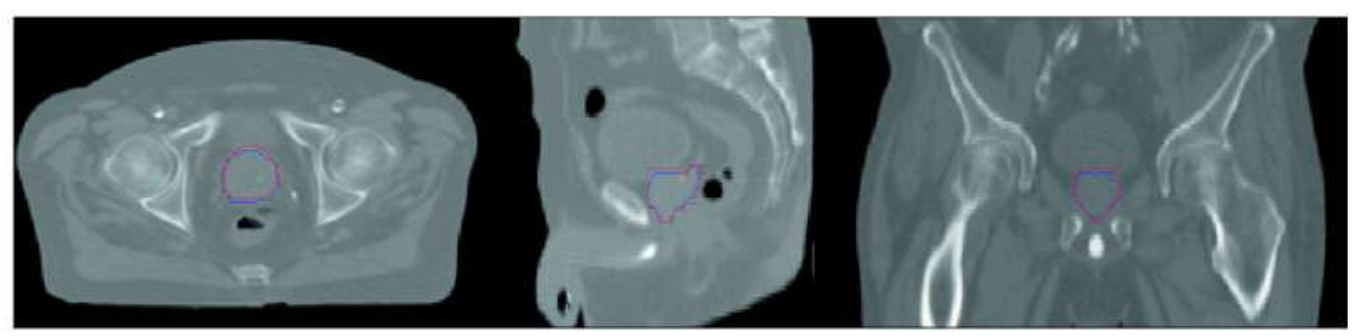

Fig. 7. Axial (left), sagittal (middle), and coronal (right) views of the study of patient 1 . The manual delineation (blue) and the automatic delineation (red) are superposed. The main inaccuracies seem to be located on the prostate-bladder interface.

the sagittal view (middle), and the coronal view (right) of the session study of the patient. The manual delineation (blue) and the automatic delineation (red) are superposed. The use of a combination of an axial, a sagittal, and a coronal view can offer a preliminary idea of the delineation similarity, but it is necessary to choose the correct slices for each view. Even then, a global view of the error distribution cannot be obtained since the visualization of the delineations is limited. The use of a single $2 \mathrm{D}$ polar map representation allows the visualization of the same information that the representation of all the axial, sagittal, and coronal views of the organ, but facilitates the analysis of the information.

In the case study, both delineations are quite close, but some differences can be detected studying the images. While both apices have quite good matching, the automatic delineation of the prostate-bladder interface and the concavity formed by the pressure of the rectum on the prostate is quite different from the reference delineation, as can be seen in different axial and sagittal views. The inaccuracies on the prostate-bladder interface are very common even comparing different manual delineations.

The results of the proposed evaluation methodology, shown in Fig. 8, allow a proper measure of the distance between the delineations, offering a quantitative evaluation of the automatic algorithm. In the case study the polar map shows a very good fit in the apex region $\left(-90^{\circ}<\varphi<-60^{\circ}\right)$ and a small error of $3 \mathrm{~mm}$ in the prostate-bladder interface $\left(30^{\circ}<\varphi<60^{\circ}\right.$ and $\left.-180^{\circ}<\theta<0^{\circ}\right)$. Nevertheless, the distance between segmentations around the seminal vesicles, and even at the concavity formed by the rectum pressure over the prostate, grows to $4-9 \mathrm{~mm}$.

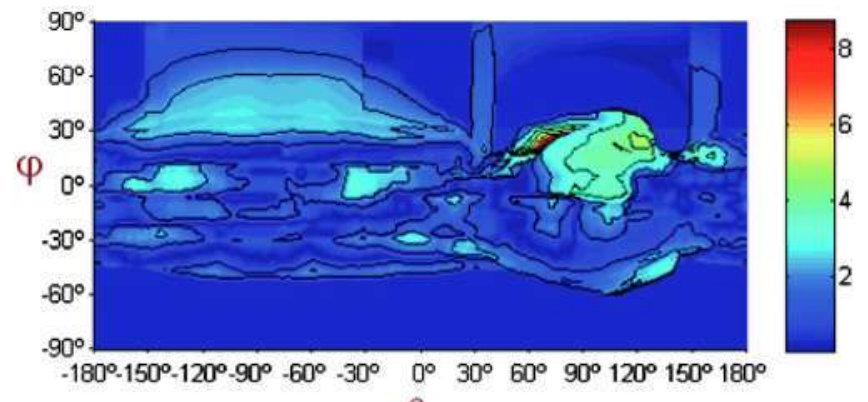
$\theta$

FIG. 8. Polar map of the error distribution in patient 1 . The polar map allows a proper measure of the distance between the delineations, offering a quantitative evaluation of the delineation algorithm.

\section{III.B. Error metric}

The error distributions obtained using our metric and the one proposed in Ref. 14 are quite similar, but the inaccuracies on the modeled concavities result in some differences, as was explained in Sec. II B.

The polar map of Fig. 9 shows the distribution and magnitude of the differences between our metric and the metric proposed in Ref. 14. These differences affect more than $2 \%$ of the polar map, and concentrate around the concavities near the seminal vesicles and the apex as shown before in Fig. 5 . Moreover, the maximum value is over $4 \mathrm{~mm}$, almost half of the maximum error value in the case study.

These results imply that our proposed metric detect errors in the delineation's matching at some control directions that previous metrics classify as a perfect matching.

\section{III.C. Volume reconstruction}

The influence of the volume reconstruction algorithm on the final results is limited because the evaluation of a registration algorithm is based on the sum of various individual cases, and the small errors are smoothed. If the volume reconstruction algorithm is able to model the concavities of the organ, such as the marching cubes algorithm or the marching tetrahedrons algorithm, the error distribution is quite similar in both cases. Figure 10 shows the average error distribution using the Origami algorithm (left) and using the marching tetrahedrons algorithm (right) over all the cases of study. The differences between error distributions are below $2 \mathrm{~mm}$, and

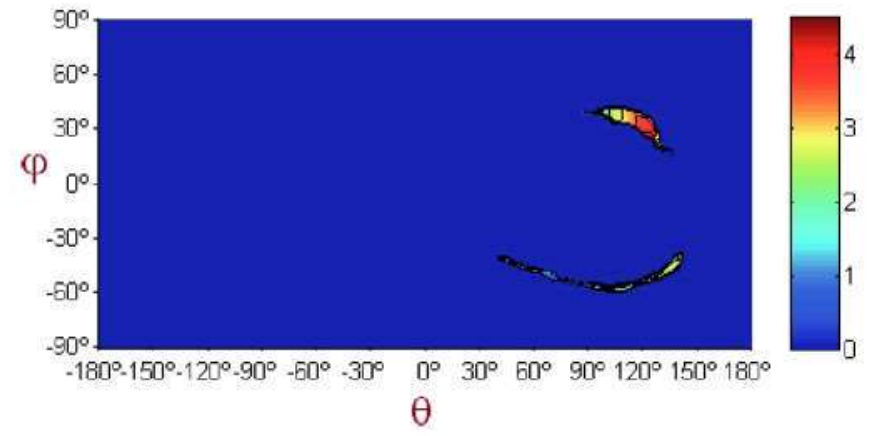

FIG. 9. Polar map of the differences between the proposed metric and the metric proposed by Mageras (Ref. 14). These differences affect more than $2 \%$ of the polar map and are concentrated around the concavities. 

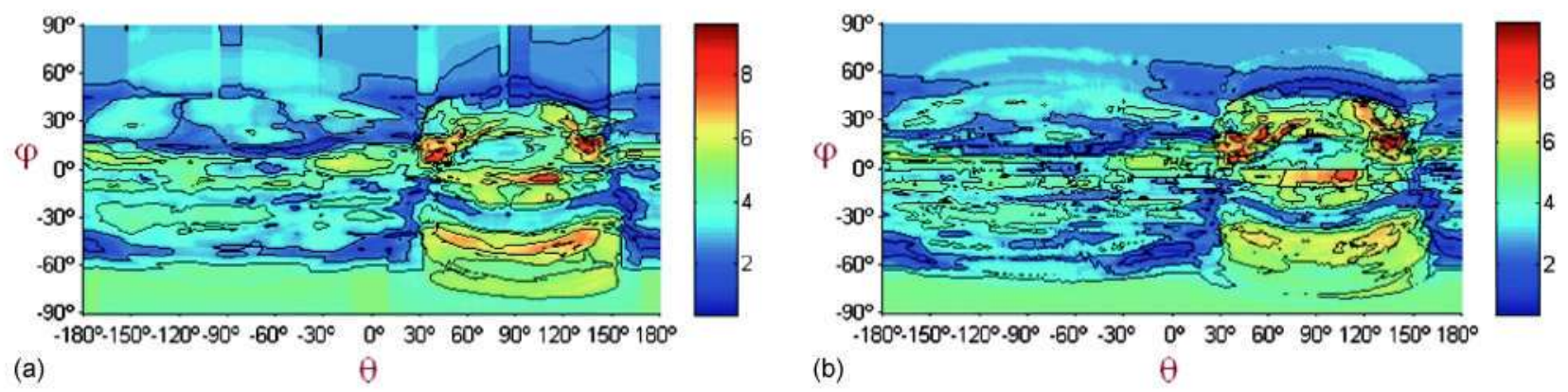

FIG. 10. (a) Polar map of the average error distribution using the Origami algorithm. (b) Polar map of the average error distribution using the marching tetrahedrons algorithm. The differences between error distributions are basically due to the stairlike reconstruction that results from the application of the marching tetrahedrons algorithm to the nonsmoothed segmented volumes.

it is basically due to the stairlike reconstruction that results from the application of the marching tetrahedrons algorithm to the nonsmoothed segmented volumes.

The main advantages that Origami offers compared to marching tetrahedrons is that the reconstructions are smoother (due to the linear interpolation between slices developed in Origami), and it is achieved faster. Marching tetrahedrons compute an evolving surface formed by hundreds of thousands of triangles, and the intersection between these triangles and a high number of control directions needs a lot of computation time and RAM resources. Origami does not compute any evolving surface, so the reconstruction time is below $0.1 \mathrm{~s}$ on average for an angular resolution of $\delta=1^{\circ}$, while marching tetrahedrons needs almost $3 \mathrm{~s}$ on average for the same case.

The results show that the main average registration errors are present around the seminal vesicles, the apex and the prostate-bladder interface. These results are consistent with the known difficulty of accurately delineating the seminal vesicles, detecting the interface between the prostate and the bladder and the location the apex.

\section{CONCLUSIONS}

The evaluation method presented in this paper provides a smooth, fast, and accurate volume reconstruction, a measure of the maximum error per control direction valid for concave and convex volumes, and a complete 3D description of nonrigid registration algorithm's performance. As a result, the evaluation methodology is a useful tool for the study and comparison of registration algorithms for adaptive radiotherapy.

Origami is a fast and accurate reconstruction algorithm that offers smooth reconstructions with a great spatial resolution in much less than a second. Nevertheless, experimental results show that the accuracy of the volume reconstruction algorithm is only relevant up to a certain extent. Due to the smoothness caused by the sum of every case, the error added by the reconstruction in each case will be negligible as long as the reconstruction algorithm is capable of modeling concavities and convexities. As a result, other reconstruction algorithms, such as marching cubes or its evolution marching tetrahedrons, will be as valid for this purpose as Origami. The metric selected for quantifying the registration error has been shown of utmost importance in a quantitative evaluation based on measuring distances between volumes. Radial distance and similar metrics are not valid for the evaluation of concave-convex volumes, distorting the error distribution and leading to an incorrect definition of the problematic regions. We have defined an error metric based on the Haussdorf-Chebysev distance that offers a consistent measure of the maximum error per control direction and improves the results of previous similar metrics.

\section{ACKNOWLEDGMENTS}

The authors will like to thank Dr. Zapatero Laborda from University Hospital of La Princesa for supplying the plan and control contours.

\footnotetext{
a)Electronic mail: brvila@ gbt.tfo.upm.es

${ }^{1} \mathrm{~W}$. Lu et al., "Deformable registration of the planning image (KVCT) and the daily images (MVCT) for adaptive radiation therapy," Phys. Med. Biol. 51, 4357-4374 (2006).

${ }^{2}$ P. Jannin, M. Fitzpatrick, D. Hawkes, X. Pennec, R. Shahidi, and M. W. Vannier, "Editorial: Validation of Medical Image Processing in ImageGuided Therapy," IEEE Trans. Med. Imaging 51(11), 1445-1449 (2002).

${ }^{3}$ J. P. W. Pluim, B. Likar, and F. A. Gerritsen, Editors, "Biomedical Image Registration, 3rd International Workshop, WBIR, 2006, Lect. Notes Comput. Sci. 4057 (2006.

${ }^{4} \mathrm{H}$. Wang et al., "Implementation and validation of a three-dimensional deformable registration algorithm for targeted prostate cancer radiotherapy," Int. J. Radiat. Oncol., Biol., Phys. 61(3), 725-735 (2005).

${ }^{5} \mathrm{P}$. E. Huber, U. Malsch, and C. Thieke, "An enhanced block matching algorithm for fast elastic registration in adaptive radiotheraphy," Phys. Med. Biol. 51(19), 4789-4806 (2006).

${ }^{6}$ M. Chen, W. Lu, Q. Chen, K. Ruchala, and G. Olivera, "ROI constrained deformable image registration for on-line adaptive radiotherapy," Int. J. Radiat. Oncol, Biol., Phys. 69(3), S727-S728 (2007).

${ }^{7} \mathrm{~J}$. Xia, Y. Chen, and S. S. Samant, "The Juggler algorithm: A hybrid deformable image registration algorithm for adaptive radiotherapy," Medical Imaging 2007: Physics of Medical Imaging, Proc. SPIE 6510, 65105J 2007.

${ }^{8}$ S. S. Samant, J. Xia, P. Muyan-Özçelik, and J. D. Owens, "High performance computing for deformable image registration: Towards a new paradigm in adaptive radiotherapy," Med. Phys. 35(8), 3546-3553 (2008).

${ }^{9} \mathrm{~J}$. West et al., "Comparison and evaluation of retrospective intermodality brain image registration techniques," J. Comput. Assist. Tomogr. 21, 554568 (1997).

${ }^{10} \mathrm{P}$. Hellier, C. Barillot, L. Corouge, B. Gibaud, G. Le Goualher, D. L. Collins, A. Evans, G. Malandain, N. Ayache, G. E. Christensen, and H. J. Johnson, "Retrospective evaluation of inter-subject brain registration," IEEE Trans. Med. Imaging 22, 1120-1130 (2003).

${ }^{11}$ G. E. Christensen, X. Geng, J. G. Kuhl, J. G. Bruss, T. J. Grabowski, I. A. Pirwani, M. W. Vannier, J. S. Allen, and H. Damasio, "Introduction to the
} 
non-tigid registration evaluation project (NIREP)," in Biomedical Image Registration. Third Internationa] Workshop, WBIR, 2006 Lect. Notes Comput. Sci. [ 4057, 128-135 (2006)].

${ }^{12} \mathrm{G}$. Getig, M. Jomier, and M. Chakos, "Valmet: A new validation tool for assessing and improving 3D object segmentation." in MICCAI, 2001, edited by W. J. Niessen and M. A. Viergever Lec1. Noles Comput. Sci. [ 2208, 516-528 (2001)].

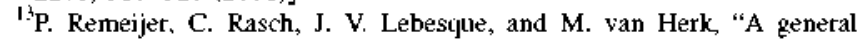
methodology for three-dimensional analysis in target volume delineaion." Med. Phys. 26(6), 931-940 (1999).

${ }^{14}$ A. Pevsner et al. ${ }^{+4}$ Evaluation of a deformable inmage matching method for quantifying lung modion in tespitation-correlated CT images," Med.
Phys. 33(2), 369-376 (2006.

${ }^{15}$ M. Foskey, B. C. Davis. L. Goyal, S. Chang. E. Chaney, N. Strehl. S. Tomei. J. Rosentnan, and $S$. Joshi, "'Large deformalion 3D inage registration in image-gnided radiation therapy," Phys. Med. Biol. 50. 5869$5892(2005)$.

${ }^{16}$ F. Gaya, B. Rodriguez-Vila. F. del Pozo. F. Garcia-Vicente, and E. Gomez, ${ }^{+} \mathrm{A}$ new Inethod for quantitative evaluation of target volume variations in radiotherapy planning," in Proceedings of the Fourth European Conference of the International Federation for Medical and Biomedical Engineering. Antwerp. Belgium. 2008. pp. 526-529 (unpublished).

${ }^{17} \mathrm{~J}$. P. Thirion, "Image matching as a diffusion process: An analogy with Maxwell's demons," Med. Image Anal. 2(3), 243-260 (1998). 